\title{
Prevalence of Metallo-Beta-Lactamase among Carbapenem Resistant Pseudomonas aeruginosa Strains Isolated from Patients Attending a Teaching Tertiary Care Hospital in Tamilnadu
}

Nithyalakshmi Jayakumar ${ }^{1}$, Mohanakrishnan Kandasamy ${ }^{2}$, Ravinder Thygarajan ${ }^{3}$, Sumathi Gnanadesikan ${ }^{4}$, Anitha S. ${ }^{5}$

1, 2, 4,5 Department of Microbiology, Sri Muthukumaran Medical College Hospital and Research Institute, Affiliated to Tamilnadu Dr. MGR Medical University, Chikkarayapuram, Mangadu, Chennai, Tamil Nadu, India. ${ }^{3}$ Department of Microbiology, Madras Medical College, Affiliated to Tamilnadu Dr. MGR Medical

University, Chennai, Tamil Nadu, India.

\section{ABSTRACT}

\section{BACKGROUND}

Infections due to carbapenem resistant Pseudomonas aeruginosa (CRPA) are considered a threat to public health and identifying the mechanism of resistance is important to achieve successful therapeutic outcomes. The objectives of the present study were to determine the prevalence of carbapenem resistant $P$. aeruginosa in our hospital settings, identify the antimicrobial susceptibility pattern of CRPA and carbapenem sensitive Pseudomonas aeruginosa (CSPA) isolates, detect the prevalence of multiple drug-resistant (MDR) P. aeruginosa among CRPA isolates and identify the prevalence of metallo-beta-lactamase (MBL) producing P. aeruginosa among the CRPA isolates.

\section{METHODS}

This is a prospective, descriptive study. We investigated all samples received in our clinical microbiology laboratory during the study period of one year from Feb 2018 to Jan 2019. Samples showing growth of Pseudomonas aeruginosa were included in the study. Antimicrobial susceptibility testing of the isolates was done by Kirby-Bauer disc diffusion method on Mueller-Hinton agar and isolates were identified as MDR and CRPA according to Clinical and Laboratory Standards Institute, $2019^{3}$ (CLSI) guidelines. CRPA isolates were further subjected to phenotypic detection of MBL by combined disc test (CDT).

\section{RESULTS}

The total number of Pseudomonas aeruginosa isolates was 134. Antimicrobial susceptibility test identified 29 (21.64\%) and 105 (78.35\%) isolates as CRPA and CSPA, respectively. CRPA isolates were recovered from the respiratory tract $(\mathrm{n}=11$, $37.93 \%)$, urine ( $n=9,31.03 \%)$, ear swab $(n=4,13.79 \%)$, blood $(n=3,10.34 \%)$ and pus $(\mathrm{n}=2,6.89 \%)$. We observed that resistance rate of CRPA was significantly higher as compared to that of CSPA. Percentage prevalence of MDR-PA among CRPA isolates was $51.7 \%(\mathrm{n}=15) .29 \mathrm{CRPA}$ isolates were subjected to combined disc test and majority $24(82.75 \%)$ of them were found to be MBL producers.

\section{CONCLUSIONS}

Detection of MBL should be a routine practice. This early detection not only helps the clinicians to optimise the antibiotics in an effective way but also prevents dissemination of these isolates.

\section{KEY WORDS}

MBL, Pseudomonas aeruginosa, CRPA, Resistance Profile, Imipenem resistant Pseudomonas aeruginosa
Corresponding Author: Dr. Nithyalakshmi Jayakumar, Professor, Department of Microbiology, Sri Muthukumaran Medical College Hospital and Research Institute,

(Affiliated to Dr. MGR Medical University), Chikkarayapuram,

Mangadu, Chennai - 600069,

Tamil Nadu, India.

E-mail: nithya.smmcri@gmail.com

\section{DOI: $10.14260 /$ jemds/2020/867}

How to Cite This Article:

Jayakumar $N$, Kandasamy $M$, Thygarajan $R$, et al. Prevalence of metallo-beta-lactamase among carbapenem resistant pseudomonas aeruginosa strains isolated from patients attending a teaching tertiary care hospital, in Tamilnadu. J Evolution Med Dent Sci 2020;9(52):3968-3972, 10.14260/jemds/2020/867 DOI:

Submission 14-08-2020,

Peer Review 03-10-2020,

Acceptance 11-10-2020,

Published 28-12-2020.

Copyright (C) 2020 JEMDS. This is an open access article distributed under Creative Commons Attribution License [Attribution 4.0 International (CC BY 4.0)] 


\section{BACKGROUND}

In the recent times, there is dramatic increase in the prevalence of infections caused by multidrug resistant $P$. aeruginosa worldwide. Carbapenems such as imipenem or meropenem are the cornerstone in the treatment of such infections. Unfortunately, infections due to carbapenem resistant Pseudomonas aeruginosa (CRPA) have been identified and reported worldwide. These infections are considered as public health crisis as it limits the therapeutic choice and often associated with high mortality.

Development of carbapenem resistance in P. aeruginosa may be due to alterations in outer membrane permeability (loss of porin channels-OprD), upregulation of efflux pump (MexA-MexB-OprM) or by the production of class B betalactamases, Metallo-beta-lactamase. ${ }^{1}$

MBLs are designated in class $\mathrm{B}$ under Ambler classification, requires the metal cofactor for its catalytic activity (Zn). It hydrolyses almost all beta-lactams including cephalosporins, penicillins and carbapenems except monobactams. It is inhibited by (ethylenediaminetetraacetic acid) EDTA and thiol compounds. Genes encoding MBL in $P$. aeruginosa are located on integron class 1 or plasmids which confers not only their ability to spread to other strains but also to different genera like Acinetobacter or Enterobacteriaceae. This can have an impact on increasing the burden of resistant strains. Early detection is of at most important to reduce their spread and select appropriate antibiotics. ${ }^{2}$

\section{Objectives}

- $\quad$ To determine the prevalence of carbapenem resistant $P$. aeruginosa (CRPA) in our hospital settings.

- To identify the antimicrobial susceptibility pattern of CRPA and CSPA solates.

- $\quad$ To detect the prevalence of MDR P. aeruginosa among CRPA isolates.

- To identify the prevalence of MBL producing $P$. aeruginosa among the CRPA isolates.

\section{METHODS}

This is a descriptive, prospective study conducted at clinical microbiology laboratory of a teaching tertiary care hospital and research institute for a period of 1 year. Institutional ethical committee approval was obtained. Samples such as pus, urine, blood, sputum, tracheobronchial aspirate, bronchoalveolar lavage, throat swab, ear swab, wound swab and different body fluids received during the study period were analysed.

Blood agar and MacConkey agar plates were inoculated with sample and incubated at $37 \mathrm{C}$ for $24-48 \mathrm{hrs}$. $P$. aeruginosa was identified by Gram staining morphology, colony characteristics, motility, catalase and oxidase test, growth at $42^{\circ} \mathrm{C}$ and confirmed by standard microbiological procedures.

\section{Inclusion Criteria}

Samples showing growth of Pseudomonas aeruginosa was included in the study.

\section{Antimicrobial Susceptibility Testing}

Antimicrobial susceptibility testing of the isolates was done by Kirby-Bauer disc diffusion method on Mueller-Hinton agar according to CLSI guidelines. Following antipseudomonal antibiotic discs were used.

Gentamicin (10 $\mu \mathrm{g})$, amikacin (30 $\mu \mathrm{g})$, ciprofloxacin $(5 \mu \mathrm{g})$, levofloxacin $(5 \mu \mathrm{g})$, ceftazidime $(30 \mu \mathrm{g})$, cefepime $(30 \mu \mathrm{g})$, ampicillin $(10 \mu \mathrm{g})$, piperacillin $(100 \mu \mathrm{g})$, piperacillintazobactam $(100 \mu \mathrm{g} / 10 \mu \mathrm{g})$, aztreonam $(30 \mu \mathrm{g})$, imipenem $(10 \mu \mathrm{g})$ and meropenem $(10 \mu \mathrm{g})$. The zones of inhibition were interpreted as per CLSI guidelines.

ATCC 27853 - Pseudomonas aeruginosa was used as the control organism for antibiotic sensitivity. Minimum inhibitory concentration (MIC) of polymyxin and colistin was determined using $\mathrm{E}$ test and the elliptical zone of inhibition intersecting with MIC strip was interpreted as per CLSI guidelines (2019).

\section{MDR}

Isolates showing resistance to one antimicrobial agent in three different categories of antimicrobial agents are described as multiple drug-resistant strains.

\section{CRPA}

Those isolates showing resistant or intermediate zone of inhibition to imipenem or meropenem was considered as carbapenem resistant Pseudomonas aeruginosa and sensitive as carbapenem sensitive Pseudomonas aeruginosa. Susceptibility pattern was analysed for CRPA and CSPA retrospectively.

\section{Screening}

All CRPA isolates were taken as screening positive and considered as probable MBL producers and subjected to confirmatory test by combined disc test.

\section{Combined Disc Test}

3 - 5 well isolated colonies of CRPA isolates were selected and added to peptone water to match 0.5 McFarland turbidity standard. This is inoculated on Mueller-Hinton (MH) agar plate using cotton swab. Imipenem and imipenem-EDTA discs were placed on the surface of the agar at a distance of $20 \mathrm{~mm}$ from each other. The inhibition zones displayed around the IPM and the IPM-EDTA discs were compared after 14 to $16 \mathrm{hrs}$ of incubation at $35^{\circ} \mathrm{C}$. The difference of $\geq 7 \mathrm{~mm}$ between the inhibition zone diameter of the IPM-EDTA disc and that of IPM only disk was considered to be positive for the presence of MBLs. The procedure was repeated twice to ensure the reproducibility of results ${ }^{4}$ (Figure 1 ).

\section{Statistical Analysis}

Fischer's exact two-sided test was used to compare the resistant profile of CRPA and CSPA. All $p$ values $<0.05$ were considered as statistically significant. 


\section{RESULTS}

A total of 134 non-repetitive $P$. aeruginosa isolates were recovered of which 69 (51.49\%) were from male patients and 65 (48.5) were from female patients. The age distribution of patients was as follows: less than 20 years ( $\mathrm{n}=12,8.95 \%), 20$ - 40 years $(n=26,19.41 \%), 41-60$ years $(n=60,44.77 \%)$ and $>60$ years $(n=36,26.86 \%)$.

Isolates were obtained from respiratory tract $(\mathrm{n}=57$, $42.53 \%)$, urine ( $\mathrm{n}=39,29.1 \%)$, wound swab $(\mathrm{n}=19,14.17$ $\%)$, blood ( $n=8,5.97 \%)$ ear swab $(n=6,4.47 \%)$, pus $(n=3$, $2.23 \%)$, and body fluids ( $\mathrm{n}=2,1.49 \%)$.

\section{Antimicrobial Susceptibility Profile}

Antimicrobial susceptibility test identified 29 (21.64\%) and 105 (78.35 \%) isolates as CRPA and CSPA, respectively. The resistant rates for carbapenem including imipenem and meropenem were $20.14 \%(n=27)$ and $17.91 \%(n=24)$ respectively. CRPA were recovered from the respiratory tract ( $\mathrm{n}=11,37.93 \%)$, urine $(\mathrm{n}=9,31.03 \%)$, ear swab $(\mathrm{n}=4,13.79$ $\%)$, blood ( $n=3,10.34 \%)$ and pus $(n=2,6.89 \%)$ (Table 1)

Resistant profile of CRPA for beta-lactams were ceftazidime $75.86 \%(n=22)$, piperacillin $65.5 \%(n=19)$, piperacillin / tazobactam $44.8 \%(\mathrm{n}=13)$, fluoroquinolones were ciprofloxacin $58.6 \%(\mathrm{n}=17)$, levofloxacin $51.7 \%(\mathrm{n}=$ 15), aminoglycosides were amikacin 37.93 ( $\mathrm{n}=11$ ), gentamicin $62.06 \%(n=18)$, tobramycin $58.6 \%(n=17)$. We observed resistant rate of CRPA is significantly high compared to CSPA. All isolates were $100 \%$ susceptible to polymyxin and colistin. (Table 2). Among the CRPA isolates, percentage of MDR-PA was $51.7 \%(\mathrm{n}=15)$.

\section{Prevalence of Metallo-Beta-Lactamase (MBL) Production}

Susceptibility profile of Pseudomonas aeruginosa to carbapenem including imipenem and meropenem revealed three phenotypes. imipenem resistant and meropenem resistant (IRMR) $(\mathrm{n}=22)$, imipenem resistant meropenem sensitive (IRMS) $(n=5)$ and imipenem sensitive meropenem resistant (ISMS) $(\mathrm{n}=2)$.

In total, 29 isolates were resistant to carbapenem accounting for $21.64 \%$. All of them were subjected to combined disc test and $24(82.75 \%)$ were found to be MBL producers (Figure 1). Among the MBL producers 22 were found to be both imipenem and meropenem resistant and 2 isolates were imipenem resistant meropenem sensitive.

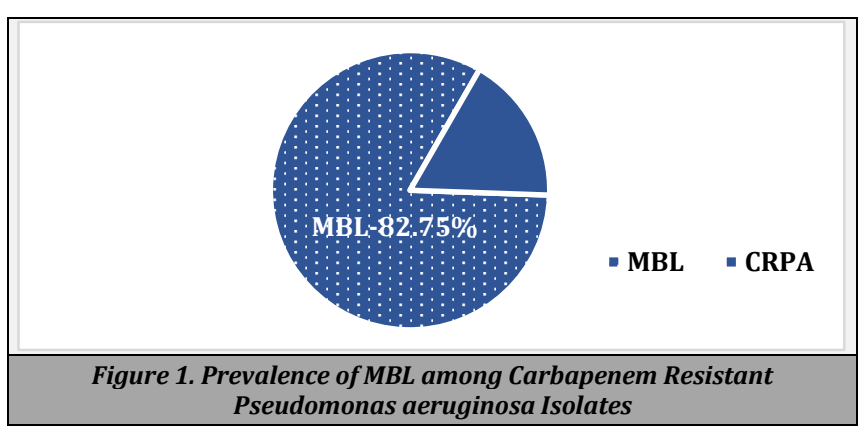

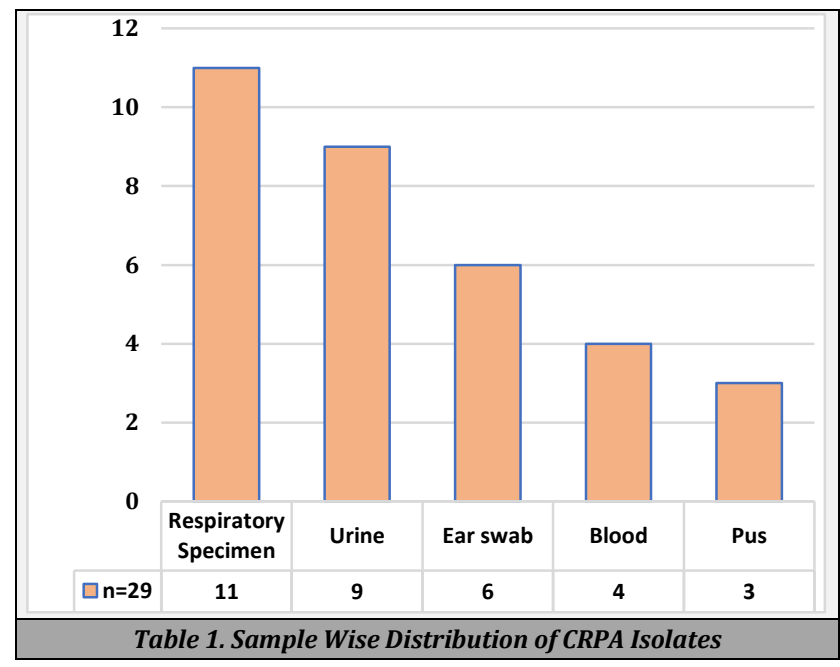

\begin{tabular}{|cccccc|}
\hline \multicolumn{1}{c}{ CRPA } & CSPA & CRPA & CSPA & P \\
R (n= 29) & R (n= 105) & S (n= 29) & S (n= 105) & Value \\
Amikacin & $11(37.93 \%)$ & $3(2.85 \%)$ & $18(62.06 \%)$ & $102(97.14 \%)$ & 0.0001 \\
Gentamicin & $18(62.06 \%)$ & $4(3.8 \%)$ & $11(37.93 \%)$ & $101(96.19 \%)$ & 0.0001 \\
Ciprofloxacin & $17(58.62 \%)$ & $3(2.85 \%)$ & $12(41.37 \%)$ & $102(97.14 \%)$ & 0.0001 \\
Levofloxacin & $15(51.72 \%)$ & $6(5.71 \%)$ & $14(48.27 \%)$ & $99(94.28 \%)$ & 0.0001 \\
Piperacillin & $19(65.51 \%)$ & $4(3.8 \%)$ & $10(34.48 \%)$ & $101(96.19 \%)$ & 0.0001 \\
Piperacillin - & $13(44.82 \%)$ & $2(1.9 \%)$ & $16(55.17 \%)$ & $103(98.09 \%)$ & 0.0001 \\
Tazobactam & $22(75.86 \%)$ & $7(6.66 \%)$ & $07(24.13 \%)$ & $98(93.33 \%)$ & 0.0001 \\
\hline Ceftazidime & Table 2. Comparison of Antimicrobial Susceptibility \\
\hline \multicolumn{5}{c}{ Profile of CRPA and CSPA } \\
\hline
\end{tabular}

\section{DISCUSSION}

Carbapenems are the reserved drugs for multidrug resistant $P$. aeruginosa. It can develop resistance to carbapenem group of antibiotics by altered outer membrane permeability, active efflux pump or by the production of metallo-beta-lactamases (MBL). In addition, acquired resistance through plasmid is common in nosocomial isolates. Spread of this resistance could be prevented by early identification. Global emergence of CRPA has been considered as public health crisis.

In our study prevalence rate of CRPA is $21.64 \%$. Our findings are in accordance with similar studies in different parts of India such as East India, ${ }^{5}$ Chennai, ${ }^{6}$ Telangana $^{7}$ and Puducherry $^{8}$ which reported $18.24 \%, 10 \%, 31 \%$ and $10.9 \%$ of $P$. aeruginosa were CRPA. However, resistant rate of $7.4 \%$ to $35.4 \%$ was reported in $\mathrm{USA}^{9}$ and $14.6 \%$, in Taiwan ${ }^{10}$ in a similar study. This could be due to the fact that prevalence of CRPA in any hospital settings depend on factors such as carrier rate among the hospital personnel, usage of broad-spectrum antibiotics, patient age and specimen source. Prior antibiotic exposure may be more important risk factors for developing carbapenem resistant infections rather than prior receipt of carbapenems. Hence, variations in prevalence with the geographic areas, emphasis the need for continuous surveillance for such isolates.

CRPA isolates were obtained from respiratory tract, urine, ear swab, blood and pus specimens. The highest percentage of CRPA $(n=11)$ was from respiratory tract. This was in contrast to Khorvash F et al. ${ }^{11}$ and Dogonchi AA et al.,12 who reported urinary tract infections are the common type of infection due to CRPA in their similar studies. This variation in type of infections associated with CRPA, implied that CRPA could be 
suspected in all specimens irrespective of type of infections. As CRPA often associated with serious healthcare-associated infection in critically ill patients, timely detection can reduce the mortality.

The resistant rates for carbapenem including imipenem and meropenem were $20.14 \%(n=27)$ and $17.91 \%(n=24)$ respectively. This is alarming sign as carbapenem are the drug of choice. Mohammed Ansar Qureshi et al. in his research has also identified such discrepant report for imipenem and meropenem with high rate of imipenem $(45.83 \%)$ and meropenem (54.16\%) resistance. ${ }^{13}$ We identified three phenotypes such as IRMR, IRMS and ISMR, this could be explained by the fact that mechanism of resistance to imipenem and meropenem are different. Emergence of such phenotypes may be due to selective antibiotic pressure exerted by injudicious use of carbapenems. Hence, in spite of being same group of antibiotics (imipenem and meropenem) susceptibility pattern of single agent should not be extrapolated for the other.

We observed the resistant profile of CRPA to antipseudomonal antibiotics tested was significantly high compared to CSPA. All of them were susceptible to polymyxins and colistin, making them ideal choice of drug for CRPA infections.

$51.17 \%$ of CRPA was found to be multidrug resistant according to the criteria defined earlier. This result was in agreement with the findings of Deanna J et al., who reported $65 \%$ of CRPA was MDR. ${ }^{14}$ This can be explained by two factors in CRPA-accumulation of several chromosomal mutations or transferrable resistant determinants encoding MBL are cotransferred with genes, aminoglycoside modifying enzymes conferring multidrug resistance.

Among CRPA isolates, 82.75 \% were MBL producers. We observed that in our settings the predominant mechanism of carbapenem resistant being production of class B metallobeta-lactamase (MBL). Similar results have been reported by Amudham et al., who found $80.4 \%$ MBL among CRPA. ${ }^{15}$ However, Dogonchi et al. in his similar study at Iran reported low prevalence of $20 \%$ MBL in CRPA. ${ }^{12}$

This suggest that prevalence varies with geographic location and understanding about the prevalence of mechanism of resistance is crucial as it guide the choice of resistance detection methods.

\section{CONCLUSIONS}

Carbapenems are the last line of antibiotics against MDR $P$. aeruginosa. We identified carbapenem resistance to be predominantly mediated by production of MBL. As detection is important to prevent their spread, identification, right choice of antibiotics, phenotypic detection methods should be routinely practiced.

Data sharing statement provided by the authors is available with the full text of this article at jemds.com.

Financial or other competing interests: None.

Disclosure forms provided by the authors are available with the full text of this article at jemds.com.

\section{REFERENCES}

[1] Sheikh AF, Rostami S, Jolodar A, et al. Detection of metallobeta lactamases among carbapenem-resistant Pseudomonas aeruginosa. Jundishapur J Microbiol 2014;7(11):e12289.

[2] Khorvash F, Yazdani M, Shabani S, et al. Pseudomonas aeruginosa - producing Metallo - $\beta$ - lactamases (VIM, IMP, SME and AIM) in the clinical isolates of intensive care units, a University Hospital in Isfahan, Iran. Adv Biomed Res 2017;6:147.

[3] CLSI. Performance standards for antimicrobial susceptibility testing. 29th edn. CLSI Supplement M100. Wayne, PA: Clinical and Laboratory Standards Institute; 2019.

[4] Yong D, Lee K, Yum JH, et al. Imipenem-EDTA disk method for differentiation of metallo- $\beta$-lactamase producing clinical isolates of pseudomonas spp. and acinetobacter spp. J Clin Microbiol 2002;40(10):3798-801.

[5] Verma N, Prahraj AK, Mishra B, et al. Detection of carbapenemase-producing Pseudomonas aeruginosa by phenotypic and genotypic methods in a tertiary care hospital of East India. J Lab Physicians 2019;11(4):28791.

[6] Porwal R, Gopalakrishnan R, Rajesh NJ, et al. Carbapenem resistant gram-negative bacteremia in an Indian intensive care unit: a review of the clinical profile and treatment outcome of 50 patients. Indian J Crit Care Med 2014;18(11):750-3.

[7] Handa D, Noman SS. Prevalence of carbapenemase producing Pseudomonas aeruginosa in ICU patients. International Journal of Scientific Research 2019;8(6):512.

[8] Shashikala, Kanungo R, Srinivasan S, et al. Emerging resistance to carbapenems in hospital acquired pseudomonas infection: a cause for concern. Indian J Pharmacol 2006;38(4):287-8.

[9] Morrow BJ, Pillar CM, Deane J, et al. Activities of carbapenem and comparator agents against contemporary US Pseudomonas aeruginosa isolates from the CAPITAL surveillance program. Diagn Microbiol Infect Dis 2013;75(4):412-6.

[10] Lin KY, Lauderdale TL, Wang JT, et al. Carbapenemresistant Pseudomonas aeruginosa in Taiwan: prevalence, risk factors and impact on outcome of infections. J Microbiol Immunol Infect 2016;49(1):52-9.

[11] Khorvash F, Yazdani M, Shabani S, et al. Pseudomonas aeruginosa-producing metallobeta lactamases (VIM, IMP, SME and AIM) in the clinical isolates of intensive care units, a University Hospital in Isfahan, Iran. Adv Biomed Res 2017;6:147.

[12] Dogonchi AA, Ghaemi EA, Ardebili A, et al. Metallo- $\beta$ lactamase-mediated resistance among clinical carbapenem-resistant Pseudomonas aeruginosa isolates in Northern Iran: a potential threat to clinical therapeutics. Ci Ji Yi Xue Za Zhi 2018;30(2):90-6.

[13] Qureshi MA, Bhatnagar RK. Phenotypic characterisation of ESBL, AmpC and MBL producers among the clinical isolates of multidrug resistant Pseudomonas aeruginosa. Int J Curr Microbiol App Sci 2016;5(10):749-58. 
[14] Buehrle DJ, Shields RK, Clarke LG, et al. Carbapenemresistant Pseudomonas aeruginosa bacteremia: risk factors for mortality and microbiologic treatment failure. Antimicrob Agents Chemother 2016;61(1):e01243-16.
[15] Amudhan MS, Sekar U, Kamalanathan A, et al. bla (IMP) and bla (VIM) mediated carbapenem resistance in pseudomonas and acinetobacter species in India. J Infect Dev Ctries 2012;6(11):757-62. 